\title{
Prevention of Progression from Prediabetes to Diabetes in Hypogonadal Men Treated with Testosterone for up to 12 Years
}

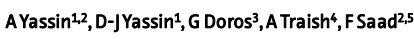

Gulf Institute for Urology and Andrology, Norderstedt, Germany

\begin{abstract}
Background
Hypogonadism is a predictor of type 2 diabetes mellitus (T2DM) (Laaksonen et al. Diabetes Care 2004; 27:1036-1041).

To study long-term effectiveness and safety of testosterone undecanoate injections (TU) in hypogonadal men from a registry study in a urological institute.

In a real-life, ongoing, cumulative, observational registry study, 505 hypogonadal men with total testosterone levels $\leq 12 \mathrm{nmol} /$ were enrolled at the time of analysis.

$253(50.1 \%)$ had prediabetes at baseline, defined by $\mathrm{HbA}_{1 \mathrm{C}}$ between 5.7 and $6.4 \%$, according to ADA definition. 175 received treatment with TU 3-monthly injections up to 12 years (T-group) following an initial 6-week interval. In 76/175 men,

testosterone treatment had been temporarily interrupted for a mean of 16 months, due to reimbursement problems. All men continued testosterone therapy after this intermission period. 78 patients opted against testosterone therapy and served as controls (CTRL). At each or each other visit, anthropometric and metabolic parameters were measured.

Mean changes over time between groups were compared by mixed effects model for repeated measures with random effect for intercept and fixed effects for time, group and their interaction and adjusted for age, weight, waist circumference, fasting

glucose, blood pressure and lipids. Mean age at baselinew 5 the T-group, 68 in CTRL. $5.9 \pm 0.2$ to $6.2 \pm 0.6 \%$ after 8 years $(p<0.0001)$. The mean difference at 8 years was $-0.8 \%(p<0.0001)$.

Not a single patient in the T-group progressed from prediabetes to T2DM. Only 23 men (13\%) still fulfilled prediabetes criteria, 152 Mean $4.9 \pm 1.4$ to $5.0 \pm 0.9 \mathrm{mmol} / \mathrm{L}$ at 8 years. The mean difference between groups at 8 years was $-0.7(\mathrm{p}=0.0549)$.
\end{abstract}
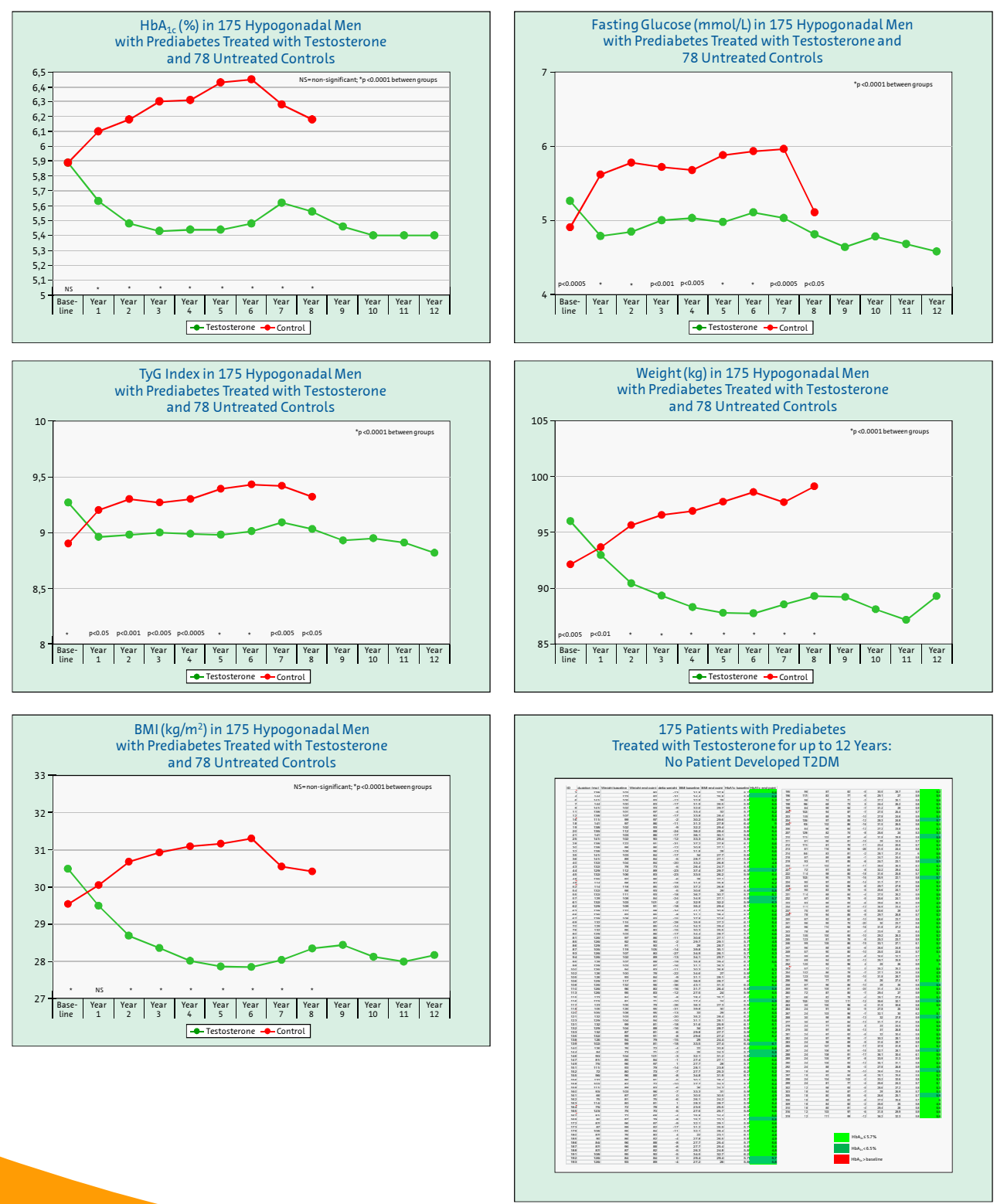

In the T-group, all injection
The triglyceride:HDL ratio, a surrogate parameter for insulin resistance, decreased in the T-group from $6.3 \pm 2.9$ to $4.4 \pm 2.3$ at 8 and $3.1 \pm 1.2$ at 12 years $(p<0.0001)$. In CTRL,

The TyG index, another surrogate parameter for insulin resistance, decreased in the T-group from $9.3 \pm 0.4$ to $9.0 \pm 0.4$ at 8 and $8.8 \pm 0.3$ at 12 years $(p<0.0001)$. In $(12$,
groups at 8 years was $-0.3(p<0.05)$.

Mean weight decreased from $96 \pm 11.7$ to $89.3 \pm 9.8$ at 8 and $87.1 \pm 7.5 \mathrm{~kg}$ at 12 years in the T-group $(\mathrm{p}<0.0001)$. In CTRL, weight increased from $92.1 \pm 9.9$ to $98.2 \pm 6.3$ at 8 years. The difference between groups at 8 years was $-14.6 \mathrm{~kg}(\mathrm{p}<0.0001)$.

Mean waist circumference decreased from $104.7 \pm 7.1$ to $98.4 \pm 6.6$ at 8 and $94.1 \pm 2.2 \mathrm{~cm}$ at 12 years in the T-group $(p<0.0001)$. In CTRL, waist circum
$-12.2 \mathrm{~cm}(\mathrm{p}<0.0001)$.

Limitations: The study was not designed to investigate effects of testosterone on prediabetes. Patients were not randomized as this was not possible in a regular medical office. There was no structured lifestyle advice. The untreated control patients were approximately 6.5 years older at baseline. The reason is that the T-group contained a number of young men with primary hypogonadism (Klinefelter's syndrome, orchiectomy after testicular cancer). However, baseline $\mathrm{HbA}_{1 \mathrm{c}}$ was equal in both groups. Strengths: The long duration of follow-up

This primary preventive effect may have been mediated by sustained weight loss in the testosterone-treated group and by the well-documented increase in lean body mass that invariably occurs when hypogonadal men are treated with testosterone.

Conclusion

Testosterone therapy prevented progression from prediabetes to T2DM in hypogonadal men while more than one third of Corterion
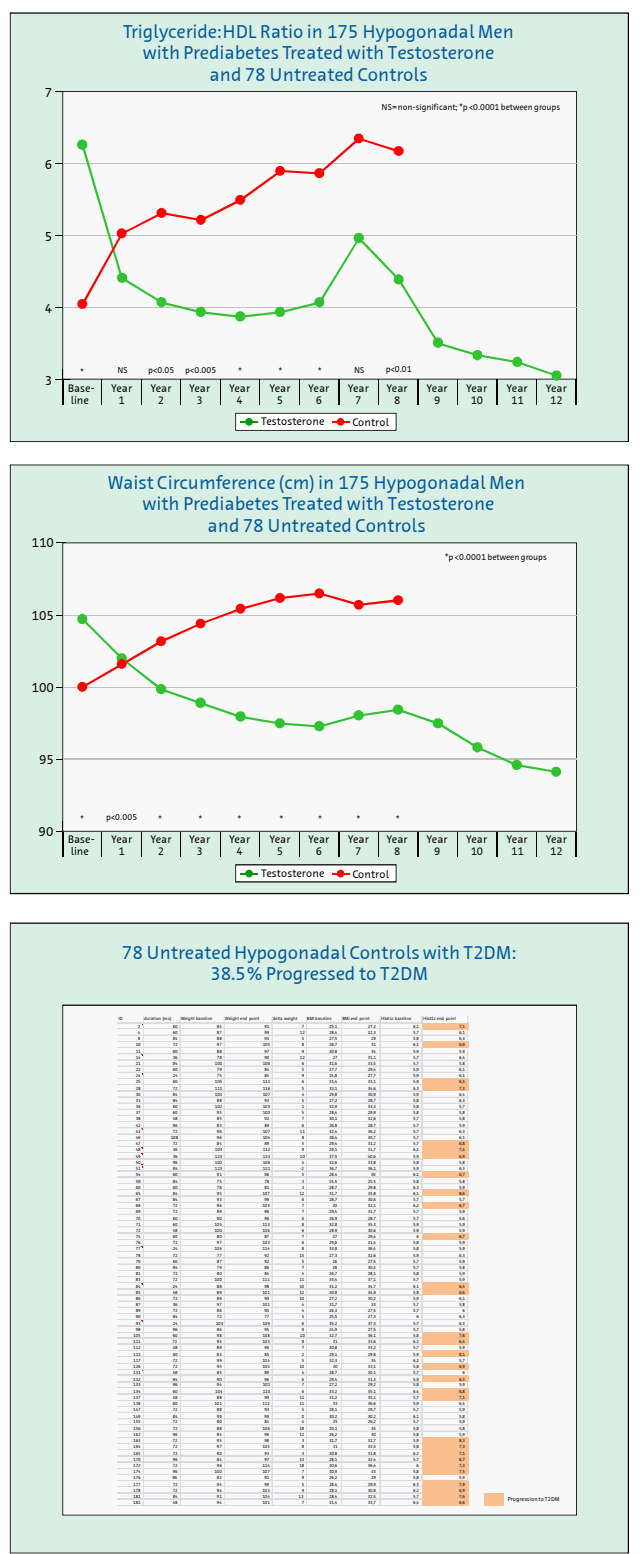